\title{
Medizinische Praxiskoordinatorin
}

\section{Bruno Gutknecht ${ }^{a}$, Ernst Gähler ${ }^{b}$}

a Fürsprecher, OdA Berufsbildung MPA,

b Dr. med., Vizepräsident der FMH, Departementsverantwortlicher Paramedizinische Berufe

Korrespondenz: Bruno Gutknecht OdA Berufsbildung MPA

Monbijoustrasse 35 CH-3011 Bern

info[at]odamed.ch
Anfang Oktober 2014 hat das Staatssekretariat für Bildung, Forschung und Innovation SBFI die Prüfungsordnung Medizinische Praxiskoordinatorin mit eidgenössischem Fachausweis im Bundesblatt publiziert. Die Medizinische Praxiskoordinatorin in den Fachrichtungen Klinische und Praxisleitende Richtung ist eine Weiterbildungsstufe für Medizinische Praxisassistentinnen (alle Informationen dazu unter www.odamed.ch).

\section{Einzig der Bund Schweizerischer Medizinischer Praxisassistentinnen BSMPA hat Einsprache erhoben.}

Die FMH gehört neben Hausärzte Schweiz, der Association Romande des Assistantes Médicales ARAM, dem Schweizerischen Verband Medizinischer PraxisAssistentinnen SVA und dem Schweizerischen Verband Medizinischer Berufsschulen SVMB der Projekt- und Prüfungsträgerschaft, der OdA Berufsbildung MPA odamed, an. In langen Verhandlungsrunden mit den umliegenden Berufsfeldern hat die OdA Berufsbildung MPA sämtliche Differenzen an den Schnittstellen der Berufsbilder (Pflege, Spitex, Ernährungsberatung, Therapien usw.) bereinigen können. In der Publikation der Prüfungsordnung hat nun einzig der Bund Schweizerischer Medizinischer Praxisassistentinnen BSMPA (bestehend aus drei kleinen MPA-Verbänden aus den Regionen Deutschschweiz, Genf und Tessin) Einsprache erhoben. Dies ist insoweit erstaunlich, als der BSMPA in zahlreichen Gesprächen versichert und auch an einer vom BAG moderierten Sitzung schriftlich zu Proto- koll gegeben hatte, keine Einwendungen und insbesondere keine Einsprache im Publikationsverfahren zu erheben.

Die Intervention des BSMPA hat zur Folge, dass das ganze Genehmigungsverfahren vorläufig gestoppt ist und eine beachtliche Zahl prüfungsbereiter MPA unter ihnen auch Mitglieder des BSMPA - im Jahr 2015 nicht wie vorgesehen zur ersten Berufsprüfung wird antreten können. Sie tragen das Zusatzrisiko,

dass die Gültigkeit ihrer mit grossem persönlichem und finanziellem Aufwand absolvierten Modulzertifikate teilweise abzulaufen droht. Zu-

dem können die von der Ärzteschaft schon seit Jahren erwarteten Tarifverhandlungen für die Abgeltung von MPA-Beratungsleistungen im Rahmen des Masterplans Hausarztmedizin solange nicht weitergeführt und abgeschlossen werden, bis keine genehmigte Prüfungsordnung mit definitivem Kompetenzprofil vorliegt.

Die Intervention des BSMPA hat zur Folge, dass das ganze Genehmigungsverfahren vorläufig gestoppt ist.

Die Einsprache des BSMPA enthält weder sachlich noch berufsbildungsrechtlich relevante Einwendungen. Sie richtet sich damit frontal gegen die Interessen der ganzen Ärzteschaft und der grossen Mehrheit der MPA in der Schweiz. Das SBFI hat das Einspracheverfahren eröffnet, wird die Parteien anhören und über die Einsprache beschliessen. Einer unterliegenden Partei steht anschliessend der Beschwerdeweg an das Bundesverwaltungsgericht offen. 